\title{
Commentaire
}

\section{Pour un portrait complet : proposition de cadre pour la surveillance et la recherche dans le domaine de la maltraitance envers les enfants au Canada}

\author{
Andrea Gonzalez, Ph. D. (1); Tracie O. Afifi, Ph. D. (2); Lil Tonmyr, Ph. D. (3)
}

(Publié en ligne le 27 septembre 2021)

Diffuser cet article sur Twitter

\section{Introduction}

La propagation rapide de la COVID-19 ainsi que la morbidité et la mortalité importantes qui lui sont associées ont entraîné une perturbation sans précédent de la vie des individus partout dans le monde. L'évolution de la pandémie et sa longue durée ont créé de l'incertitude et de l'imprévisibilité. Les multiples vagues d'infections, les hospitalisations, les décès et les restrictions imposées par les autorités de santé publique ont eu une grande incidence sur le quotidien de millions de personnes, ce qui a augmenté les risques de problèmes de santé mentale ${ }^{1-3}$. Toutefois, les données ne sont pas toujours cohérentes. En effet, selon un rapport, si la détresse psychologique a augmenté au cours des premiers mois de la pandémie, la plupart des paramètres associés à la détresse sont revenus à la normale à la mi-2020, et le sentiment de solitude, la satisfaction à l'égard de la vie et les taux de suicide sont demeurés stables ${ }^{4}$.

Ce numéro spécial souligne les effets négatifs de la première année de la pandémie sur la santé mentale et le bien-être des Canadiens. Au cours des dix premiers mois qui ont suivi le début officiel de la pandémie, les taux de consommation d'alcool et de cannabis $^{5,6}$ et de dépression ${ }^{7}$ ont augmenté, les taux de perception positive de sa santé mentale, de satisfaction à l'égard de la vie et de sentiment d'appartenance à la communauté ${ }^{8}$ ont diminué, tandis qu'aucun changement n'a été observé en ce qui concerne les idées suicidaires ${ }^{9}$.
Cependant, les Canadiens n'ont pas tous été touchés de la même manière. Comme l'affirment Varin et ses collaborateurs ${ }^{5}$, « il est essentiel de comprendre les déterminants sociaux de la santé pour élaborer des stratégies de réduction et d'atténuation des méfaits ». En effet, le fait d'être jeune, de vivre en milieu urbain et d'avoir des problèmes de santé mentale a été associé à une probabilité accrue de conséquences négatives ${ }^{5-7}$. De façon analogue, les Canadiennes, et en particulier celles qui s'occupaient d'enfants de moins de 18 ans, ont eu tendance à être plus gravement affectées, ce qui concorde avec les résultats de travaux de recherche internationaux menés sur le sujet ${ }^{3}$.

Bien qu'instructives, ces conclusions sont limitées en raison de la nature des données recueillies, des caractéristiques d'ordre sociodémographique, du caractère limité des ensembles de facteurs individuels (par exemple l'anxiété, les symptômes dépressifs, la maîtrise de soi), des facteurs communautaires (par exemple le sentiment d'appartenance) et de l'exposition aux facteurs de stress liés à la pandémie. On constate d'importantes lacunes dans notre compréhension de l'ensemble des répercussions de la COVID-19 sur les femmes et les enfants, en particulier quant à savoir si la situation a créé une "pandémie fantôme » de violence familiale, notamment de maltraitance envers les enfants.

Outre la maladie et la mort, la pandémie a entraîné un isolement social et physique, une insécurité financière, une hausse de la

\section{Points saillants}

- La pandémie de COVID-19 a entraîné une augmentation des facteurs de risque associés à la violence familiale.

- Au Canada, nous ignorons si la pandémie a exacerbé les risques de maltraitance envers les enfants.

- Les recommandations visant à renforcer notre cadre de surveillance et de recherche dans le domaine de la maltraitance envers les enfants comprennent l'ajout de questions liées à la maltraitance dans les enquêtes nationales portant sur la santé et la victimisation, par exemple dans la prochaine Enquête canadienne sur la santé des enfants et des jeunes.

- Des activités de surveillance rigoureuses et des travaux de recherche solides dans le domaine de la maltraitance envers les enfants vont fournir une source essentielle de données sur les tendances observées au fil du temps dans divers sous-groupes et nous permettre de formuler des hypothèses et de les vérifier, puis d'évaluer et de mettre en œuvre des interventions.

- En renforçant notre cadre de surveillance et de recherche dans le domaine de la maltraitance, nous aurons davantage de soutien pour nos engagements en ce qui concerne l'élimination de la violence envers tous les enfants.

Rattachement des auteurs :

1. Département de psychiatrie et des neurosciences comportementales, Université McMaster, Hamilton (Ontario), Canada

2. Département des sciences de la santé communautaire et Département de psychiatrie, Université du Manitoba, Winnipeg (Manitoba), Canada

3. Agence de la santé publique du Canada, Ottawa (Ontario), Canada

Correspondance : Andrea Gonzalez, Offord Centre for Child Studies, Université McMaster, 1280, rue Main Ouest, Hamilton (Ontario) L8S 4K1; tél. : 905-510-1652; courriel : gonzal@mcmaster.ca 
consommation d'alcool, une augmentation des problèmes de santé mentale, des fermetures d'écoles et un accès réduit aux services médicaux et sociaux, soit autant de facteurs qui ont contribué à accroître le risque de maltraitance envers les enfants. Plusieurs études confortent cette lecture de la situation ${ }^{10,11}$, tout comme les données empiriques, qui font état d'une augmentation du nombre d'appels passés à des centres d'accueil pour victimes de violence domestique et à des lignes d'assistance téléphonique pour enfants ${ }^{12}$. Cependant, il existe peu de données empiriques concernant le Canada.

Cet article recense ce que l'on sait au sujet de la violence qui a été infligée aux enfants pendant la pandémie. Il souligne les lacunes en matière de données qui existaient avant la pandémie et montre comment le fait de ne pas y remédier nuit à notre capacité de réduire les méfaits qui touchent les enfants. Les auteurs préconisent la réalisation d'activités de surveillance et de travaux de recherche de façon continue, en mettant l'accent sur les déterminants sociaux de la santé, afin d'ajuster les ressources et les efforts déployés en matière de promotion de la santé et de prévention. Cette démarche est conforme aux priorités de la ministre de la Santé et de l'Agence de la santé publique du Canada (ASPC) ${ }^{13}$. Nous proposons une approche multidimensionnelle de la surveillance et de la recherche dans le domaine de la maltraitance envers les enfants, non seulement pour remédier aux lacunes en matière de données sur le sujet pendant la pandémie, mais aussi, de façon plus générale, pour pallier les lacunes en matière de données sur la violence familiale au Canada.

\section{Maltraitance envers les enfants pendant la pandémie de COVID-19}

Bien avant la pandémie de COVID-19, la maltraitance envers les enfants était déjà reconnue comme un problème mondial s'étendant à l'ensemble du spectre sociodémographique ${ }^{14}$. Les périodes de crise, comme la pandémie actuelle et ses répercussions économiques et sociales, sont de possibles catalyseurs de violence familiale. En effet, alors que la pandémie se poursuit, on voit de plus en plus de données sur l'exposition des enfants à des formes de violence ${ }^{15}$. Si, de manière générale, les données indiquent un risque accru de victimisation, les observations sont mitigées et fluctuent en fonction de la source de la violence et du moment auquel elle survient. En fait, les cas présumés de violence faite aux enfants qui ont été dénoncés à la police ou aux services de protection de l'enfance ont diminué de jusqu'à $70 \%{ }^{12,16-18}$ dans le monde, mais les appels adressés à la police pour cause de problèmes domestiques ont augmenté de $12 \%$ au Canada ${ }^{19}$.

Les tendances en ce qui concerne les appels passés aux lignes d'assistance ne sont pas claires : selon les sources, le nombre d'appels aurait soit augmenté, soit diminué et, dans la moitié des cas, il n'y aurait eu aucun changement de tendance $^{12,19}$. Il est possible que ces variations soient dues à des différences entre les injonctions à rester à la maison, au fait que les éducateurs et autres fournisseurs de services ne voyaient plus les enfants et à l'impossibilité pour les victimes d'accéder à des services en toute sécurité ou confidentialité pendant les périodes de confinement.

En se fondant sur les dossiers d'hospitalisation, plusieurs chercheurs ont observé une augmentation des cas de blessures liées à la maltraitance chez les enfants ${ }^{12,20}$. Par exemple, le Centre hospitalier pour enfants de l'est de l'Ontario a signalé que le nombre de fractures et de traumatismes crâniens chez les enfants de moins d'un an avait plus que doublé entre septembre 2020 et janvier 2021 par rapport à la même période pendant les années ayant précédé la pandémie ${ }^{21}$. Cette observation concorde avec ce qui a été signalé au Royaume-Uni ${ }^{22}$. Des enquêtes locales menées auprès de personnes s'occupant d'enfants ont montré que les facteurs de stress liés à la pandémie, comme la perte d'emploi, l'isolement social et la détresse parentale, étaient associés à une augmentation des cas de violence émotionnelle/psychologique, de négligence physique et de supervision négligente, et à un recours accru à des pratiques disciplinaires sévères ${ }^{10-12,23}$.

Ce portrait mitigé souligne les défis persistants qui affectent la disponibilité et la qualité des données sur la violence faite aux enfants ${ }^{12,15}$. Certains de ces problèmes sont liés à un investissement insuffisant dans la réalisation d'enquêtes de routine et d'enquêtes longitudinales et dans le recours à d'autres méthodes de collecte de données pour estimer la prévalence et l'incidence de la maltraitance envers les enfants. Pour obtenir une vue d'ensemble, il convient d'associer les activités de surveillance transversale à la collecte de données longitudinales. Qui plus est, il est possible de le faire en adaptant les approches existantes dans le domaine de la recherche auprès des jeunes ${ }^{24}$. La collecte régulière de données transversales nous permet d'obtenir des estimations de prévalence au fil du temps, et les données longitudinales nous permettent de déceler des changements dans les caractéristiques de la population cible, tant au niveau du groupe qu'au niveau de l'individu.

Les systèmes de données administratives susceptibles de nous aider à estimer l'incidence à l'échelle nationale des signalements effectués auprès des autorités et des fournisseurs de services se sont révélés déficients ${ }^{15}$, les principales difficultés étant liées à un phénomène de sous-déclaration au sein du système de protection de l'enfance $^{25}$ et à des préoccupations au sujet des outils de dépistage actuellement utilisés dans les hôpitaux ${ }^{26}$. Le manque de données cohérentes et fiables a nui à notre capacité à comprendre le phénomène de la maltraitance envers les enfants en lien avec la COVID-19, à suivre les tendances en la matière et à élaborer des plans. La pandémie a révélé une déficience majeure du système, à savoir la sous-détection de l'ampleur de la maltraitance envers les enfants au Canada. Alors que nous sortons de la pandémie, nous allons avoir besoin d'une approche globale de collecte de données sur la maltraitance envers les enfants.

\section{Activités de surveillance et de recherche au Canada}

À bien des égards, le système de santé publique du Canada a fait de la question de la violence familiale une priorité, grâce au soutien de l'ensemble de la classe politique et de nombreux ministères et organismes fédéraux ${ }^{27}$. Cependant, il est possible d'en faire davantage.

Le gouvernement fédéral a ratifié et mis en œuvre la Convention de l'Organisation des Nations Unies (ONU) relative aux droits de l'enfant et a offert son soutien pour l'atteinte des objectifs du Programme de développement durable à l'horizon 2030 de l'ONU, notamment en ce qui concerne l'objectif 16.2 visant à " mettre un terme à la maltraitance, à l'exploitation et à la traite, et à toutes les formes de 
violence et de torture dont sont victimes les enfants » d'ici $2030^{28}$.

En 2018, le Canada a rejoint le Partenariat mondial pour mettre fin à la violence envers les enfants en tant que " pays pionnier », renforçant ainsi son engagement à élargir le soutien politique, à mobiliser des ressources supplémentaires et à préparer les praticiens à la lutte contre la violence faite aux enfants. Dans le cadre de cet engagement, le Canada a accepté d'accélérer les mesures prises à l'échelle nationale sur une période de trois à cinq $a n s^{28}$. Une surveillance accrue s'alignerait sur les appels à l'action de la Commission de vérité et réconciliation, qui a souligné la nécessité de publier des rapports sur les enfants des Premières nations, des Inuits et des Métis qui sont pris en charge et sur les raisons motivant leur prise en charge. Une surveillance accrue répondrait également à la recommandation de surveiller et évaluer la négligence et, indirectement, à celle de réduire le nombre d'enfants autochtones pris en charge, par l'approvisionnement de données probantes pour façonner les interventions ${ }^{29}$.

Pour déterminer si le Canada respecte son engagement en lien avec la Convention de l'ONU et les appels à l'action de la Commission de vérité et réconciliation, et pour suivre les progrès accomplis dans l'atteinte de l'objectif de développement durable visant à éliminer la violence faite aux enfants d'ici 2030, il faut mettre en place un cadre rigoureux et solide de surveillance et de recherche qui intègre de multiples sources d'information.

Dans le domaine de la santé publique, on définit habituellement la surveillance comme la collecte, l'enregistrement, l'analyse, l'interprétation et la diffusion de données en continu et systématiquement, pour éclairer et évaluer les pratiques en matière de santé publique ${ }^{30}$. Les données de surveillance sont utilisées pour faire des suivis, générer des hypothèses et analyser des différences au fil du temps entre divers sous-groupes, par exemple en fonction du sexe ou de la province et ces données sont axées sur les systèmes à une échelle plus globale. Nous avons besoin de travaux de recherche pour vérifier les hypothèses générées par les activités de surveillance et pour répondre à des questions précises concernant l'effet de causalité et l'effet du contexte. Les données probantes provenant de la surveillance et de la recherche peuvent aider à façonner la méthodologie, l'évaluation et la mise en œuvre d'interventions en santé publique et déterminer ce qui fonctionne, pour qui, et pourquoi. Cela aide les décideurs à déterminer quels sont les effets des politiques, grâce à des données utiles et disponibles au fil du temps ${ }^{31}$. À titre d'exemple de réussite, citons l'utilisation de ce type de données pour l'évaluation des programmes de formation au rôle de parent offerts au Manitoba ${ }^{32}$.

Il est possible de recueillir des données sur la violence familiale à partir de diverses sources, que ce soit les organismes de protection de l'enfance, les dossiers d'hospitalisation, les dossiers judiciaires ou les enquêtes menées auprès de la population. Dans la mesure du possible, les données doivent tenir compte des associations avec les facteurs de risque et les facteurs de protection à l'échelle individuelle, familiale, communautaire et sociale (figure 1).

La collecte de données sur la maltraitance envers les enfants repose sur une approche en santé publique en quatre étapes adoptée par l'Alliance pour la prévention de la violence. La première étape consiste en un travail de surveillance, qui permet de saisir l'ampleur et la portée du problème. La deuxième étape, celle de la recherche, vise à déterminer quels sont les facteurs de risque et les facteurs de protection. La troisième étape consiste à mettre au point des interventions et à les évaluer. La quatrième étape est celle de la diffusion à grande échelle, de l'évaluation et de la vérification continue pour veiller à ce que toutes les composantes de la stratégie concordent avec le contexte local et aient l'effet escompté, soit celui de prévenir la violence ${ }^{33}$.

Deux des possibilités d'action reconnues dans la Feuille de route du Canada pour mettre fin à la violence à l'égard des enfant ${ }^{28}$ sont liées aux principes clés de la surveillance et au plan ministériel de la ministre de la Santé13. Il s'agit 1) d'améliorer la collecte et la qualité des données ainsi que la surveillance et 2) de renforcer les données probantes sur « ce qui fonctionne » et mobiliser les connaissances.

De nombreuses initiatives ont été entreprises ou prévues pour atteindre ces objectifs. Par exemple, l'Enquête sociale générale sur la sécurité des Canadiens (menée tous les cinq ans au sein d'un échantillon représentatif à l'échelle nationale de personnes de 15 ans ou plus) comporte des questions sur une victimisation récente, sur la violence physique ou sexuelle subie au cours de l'enfance et sur l'exposition à la violence conjugale. L'Enquête sur la santé dans les collectivités canadiennes volet Santé mentale de 2012, l'Enquête sur la santé dans les collectivités canadiennes de 2019 et l'Enquête sur la sécurité dans les espaces publics et privés de 2018 comportaient des questions sur les mauvais traitements subis pendant l'enfance et sur l'exposition à la violence conjugale.

Statistique Canada collige de l'information sur la violence familiale à partir de diverses sources de données administratives, comme les rapports de police, les rapports des tribunaux de la jeunesse et les données des organismes d'aide aux victimes. L'Étude canadienne sur l'incidence des signalements de cas de violence et de négligence envers les enfants (ECI) est une initiative nationale qui vise à recueillir des données sur les enfants et les familles lorsque les services de protection de l'enfance sont impliqués en raison de cas présumés ou soupçonnés de violence ou de négligence envers les enfants ${ }^{28}$. Plusieurs cycles de cette étude ont été menés (1998, 2003 et 2008 et plus récemment en 2018/2019) sous la direction de l'Assemblée des Premières Nations (Composante Premières Nations Étude canadienne sur l'incidence des signalements de cas de violence et de négligence envers les enfants/ECI) ${ }^{28}$. Deux initiatives relativement nouvelles ont également été lancées : le Système canadien de renseignements relatifs à la protection de l'enfance et l'Ensemble minimal de données pour tous les territoires.

Bien que ces sources de données nous offrent une information précieuse, il est possible de faire mieux. Nous présentons ici diverses recommandations sur les mesures à prendre pour mettre en place un cadre solide de surveillance et de recherche au Canada.

\section{Recommandations}

1. Les enquêtes nationales devraient englober toutes les formes de maltraitance envers les enfants. À l'heure actuelle, seuls trois sous-types de maltraitance sont inclus, soit la violence physique, la violence sexuelle 
FIGURE 1

Proposition de cadre pour la surveillance et la recherche dans le domaine de la maltraitance envers les enfants

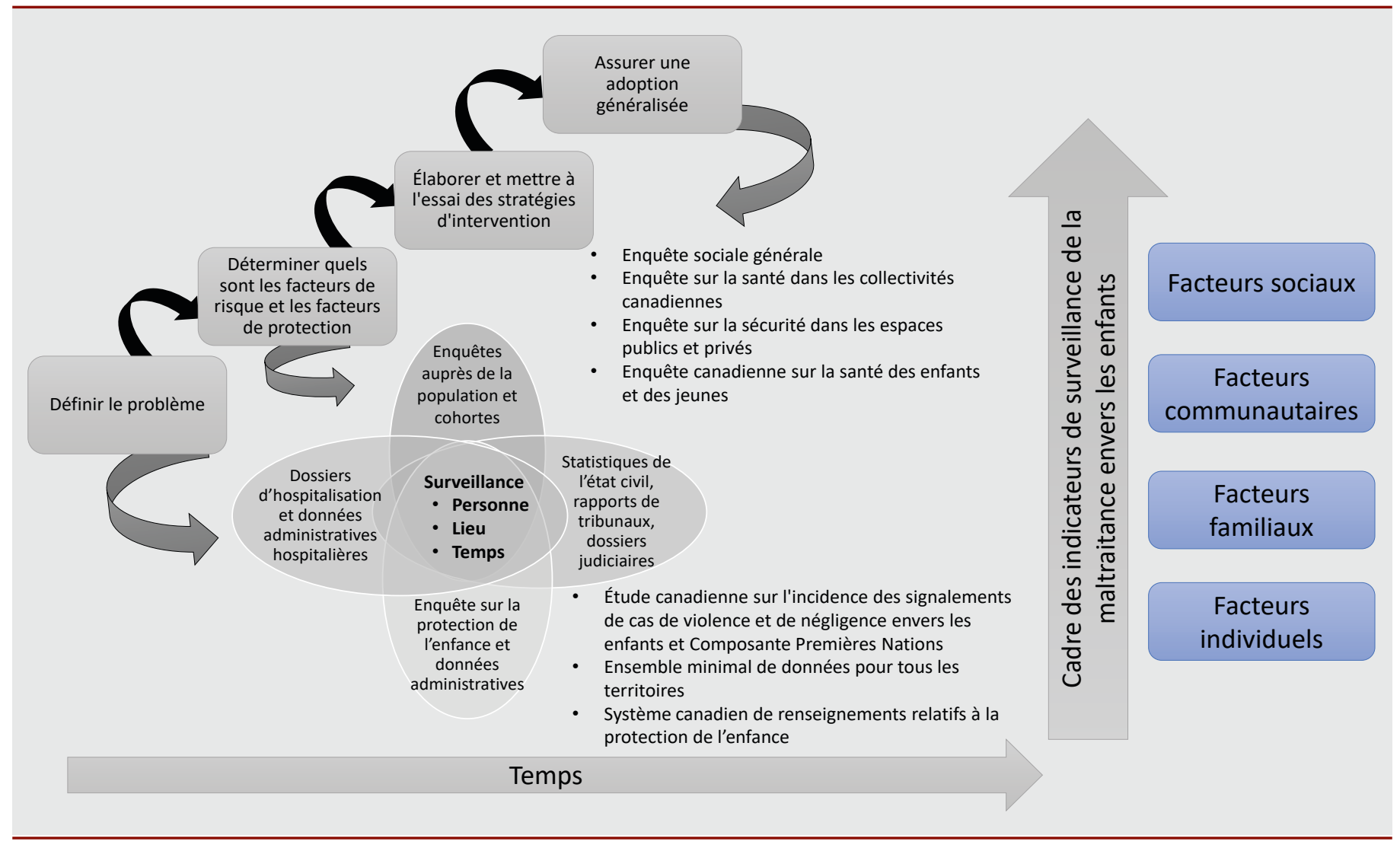

et l'exposition à la violence conjugale. Bien qu'il soit difficile de les mesurer, il faudrait également tenir compte des cas de violence psychologique et de négligence. Les questions devraient reposer sur des définitions exhaustives et bien établies et les réponses devraient pouvoir être mesurées de façon cohérente.

2. Afin qu'il soit possible de comprendre pleinement les liens entre la maltraitance envers les enfants et divers effets, toutes les enquêtes nationales axées sur la santé et la victimisation devraient comporter une série prédéterminée de questions sur la maltraitance.

3. La prochaine Enquête canadienne sur la santé des enfants et des jeunes devrait comporter des questions liées à la maltraitance envers les enfants. L'Enquête sociale générale pose de telles questions aux répondants de 15 ans ou plus, mais le Canada ne dispose pas de données nationales concernant les plus jeunes ou encore de données permettant de faire des rapprochements avec un certain nombre d'effets et de facteurs liés aux enfants.
4. Il convient de mettre au point des protocoles précis et sensibles pour recueillir de manière éthique des données sur la maltraitance envers les enfants, dans le respect des droits, de la dignité et de la sécurité des participants, et tout en respectant le devoir de signalement dans certaines situations. Une grande partie de la réticence à poser des questions sur la violence familiale découle de préoccupations relatives à la sécurité et à la possibilité de créer de la détresse. Cependant, si l'on se fie à ce qui a été observé ailleurs dans le monde, il est possible de poser des questions sur le sujet si des protocoles adéquats sont en place pla $^{34,35}$.

5. Conformément aux recommandations du Cadre des indicateurs de surveillance de la maltraitance envers les enfants $^{36}$, les données sur les facteurs de risque et les facteurs de protection multidimensionnels devraient provenir de bases de données administratives et d'enquêtes nationales.

6. Il convient également de recueillir des données longitudinales afin de comprendre l'évolution de l'état de santé et du bien-être des personnes exposées à des situations de maltraitance envers les enfants. Cela pourra se faire par des cycles successifs de collecte de données (enquêtes nationales) ou par le couplage de données administratives.

7. Dans le cas des données administratives, il faudra trouver une définition de principe bien établie et acceptable de la violence familiale pour faciliter l'extraction des données sur la maltraitance envers les enfants auprès de tous les organismes. Il devra y avoir une étroite collaboration entre les organismes responsables de la collecte de données sur la violence familiale, dans l'ensemble des secteurs et des provinces et territoires, afin que les mesures soient uniformes et que l'on puisse obtenir un portrait national de la maltraitance envers les enfants. Même si une partie de ce travail a déjà commencé un soutien et un investissement continus dans le Système canadien de renseignements relatifs à la protection de l'enfance seront nécessaires pour permettre la mise en place d'une infrastructure 
solide de gestion de données administratives sur la maltraitance.

8. Il devra y avoir des changements systémiques en matière de protection de l'enfance et un soutien plus général pour les familles qui soit axé sur la prévention primaire. Il devra également y avoir des ressources adéquates pour surveiller la mise en œuvre des interventions et pour déterminer ce qui fonctionne, pour qui, et pourquoi.

Cela peut sembler décourageant, mais nous pouvons tirer profit des expériences des autres pays. Par exemple, le Department of Justice des États-Unis, le Children's Bureau des États-Unis, les Centers for Disease Control des États-Unis et l'Australian Institute of Health and Welfare recueillent de l'information à partir de diverses sources, notamment des enquêtes sur les ménages et des données administratives officielles, dont des données de surveillance colligées par les États sur la maltraitance envers les enfants de 10 à 17 ans et sur les personnes qui s'occupent d'enfants de moins de 9 ans $^{34,35}$.

\section{Conclusion}

La violence familiale est un problème social et de santé publique urgent pour tous les Canadiens ${ }^{37}$. La pandémie de COVID-19 a révélé des lacunes considérables dans la disponibilité et la qualité des données sur la maltraitance envers les enfants au Canada. L'absence de systèmes de données de ce type a non seulement nui à notre capacité de bien comprendre les répercussions négatives de la pandémie sur les enfants et les familles, mais elle limite également notre capacité à répondre à leurs besoins en nous fondant sur des données probantes afin de les aider à se rétablir.

Cet article fait ressortir les répercussions importantes de la pandémie sur la santé mentale et le bien-être des adultes canadiens et il montre bien que ces effets ne sont pas égaux. Ce que l'on ignore, c'est si, et dans quelle mesure, la pandémie a créé une " pandémie fantôme " qui a exposé les enfants à un risque accru de maltraitance.

Selon des travaux de recherche menés à l'échelle internationale, il y a bel et bien eu une augmentation du nombre de cas de violence faite aux enfants. Même si ces hausses de cas sont relativement brèves, on peut affirmer que leurs effets seront durables étant donné que la maltraitance entraîne une multitude de conséquences à long terme sur la santé.

Alors que nous entrons dans la phase de rétablissement et de planification postpandémie, nous devons tirer parti des connaissances que nous avons acquises au sujet des lacunes de notre système et adopter un nouveau cadre pour la surveillance et la recherche dans le domaine de la maltraitance envers les enfants. Les infrastructures nécessaires à la collecte de données de surveillance et de recherche rigoureuses sur la maltraitance envers les enfants existent en partie, et il est possible de les utiliser pour accroître la faisabilité et le succès des propositions que nous formulons dans le présent commentaire. Le succès du cadre que nous proposons dépendra de la constance de l'engagement et des investissements nécessaires à la collecte et à la synthèse de données de façon continue à partir d'un certain nombre de sources, notamment des enquêtes nationales menées par Statistique Canada, des données sur les hospitalisations et blessures, des rapports de police et des rapports criminels, ainsi que des bases de données nationales sur la violence et la négligence à l'égard des enfants, comme le Système canadien de renseignements relatifs à la protection de l'enfance. Il est essentiel d'améliorer la qualité, la cohérence et la portée des données sur la maltraitance envers les enfants, non seulement pour comprendre l'ampleur de la maltraitance et surveiller les tendances en la matière, mais aussi pour respecter nos engagements envers l'atteinte de l'objectif de développement durable, soit d'éliminer la violence faite à tous les enfants.

\section{Conflits d'intérêts}

Tracie O. Afifi est rédactrice scientifique adjointe de la revue Promotion de la santé et prévention des maladies chroniques au Canada, mais elle s'est retirée du processus d'évaluation de cet article. Les auteurs déclarent n'avoir aucun conflit d'intérêts.

\section{Contributions des auteurs et avis}

AG, TOA et LT ont pris part à l'élaboration du concept et ont contribué à la conception, à la rédaction et à la révision critique du manuscrit.
Le contenu de l'article et les points de vue qui y sont exprimés n'engagent que les auteurs; ils ne correspondent pas nécessairement à ceux du gouvernement du Canada.

\section{Remerciements}

Les auteurs remercient les membres du groupe de travail sur la surveillance et la recherche dans le domaine de la violence envers les enfants pour les nombreux échanges stimulants qui ont contribué à éclairer et à façonner cet article. Les auteurs tiennent également à remercier Mary Sue Devereaux et Joanna Odrowaz pour leur aide en matière de travail éditorial.

\section{Références}

1. Alzueta E, Perrin P, Baker FC, et al. How the COVID-19 pandemic has changed our lives: a study of psychological correlates across 59 countries. J Clin Psychol. 2021;77(3):556-570. https://doi.org/10.1002/jclp.23082

2. Boden M, Zimmerman L, Azevedo $\mathrm{KJ}$, et al. Addressing the mental health impact of COVID-19 through population health. Clin Psychol Rev. 2021;85:102006. https://doi.org/10 .1016/j.cpr.2021.102006

3. Xiong J, Lipsitz O, Nasri F, et al. Impact of COVID-19 pandemic on mental health in the general population: a systematic review. J Affect Disord. 2020;277:55-64. https://doi .org/10.1016/j.jad.2020.08.001

4. Aknin LB, DeNeve JE, Dunn EW, et al. The Lancet's COVID-19 Commission Mental Health Task Force. Mental health during the first year of the COVID-19 pandemic: a review and recommendations moving forward. Perspect Psychol Sci [prépublication PsyArXiv]. 19 févr. 2021 [consultation le 31 juill. 2021]. https://doi.org/10 .31234/osf.io/zw93g

5. Varin M, Hill MacEachern K, Hussain $\mathrm{N}$, Baker MM. Mesurer les changements autodéclarés relatifs à la consommation d'alcool et de cannabis au cours de la deuxième vague de la pandémie de COVID-19 au Canada. Promotion de la santé et prévention des maladies chroniques. 2021;41(11): 357-363. https://doi.org/10.24095 /hpcdp.41.11.02f 
6. Hill MacEachern K, Venugopal J, Varin M, Weeks M, Hussain N, Baker MM. Adopter une perspective axée sur le genre pour comprendre les changements autodéclarés de la consommation d'alcool et de cannabis pendant la deuxième vague de la pandémie de COVID-19 au Canada, septembre à décembre 2020. Promotion de la santé et prévention des maladies chroniques. 2021;41(11):364373. https://doi.org/10.24095/hpcdp $.41 .11 .03 \mathrm{f}$

7. Shields M, Tonmyr L, Gonzalez A, et al. Symptômes du trouble dépressif majeur pendant la pandémie de COVID-19 : résultats obtenus à partir d'un échantillon représentatif de la population canadienne. Promotion de la santé et prévention des maladies chroniques. 2021;41(11):374-393. https:// doi.org/10.24095/hpcdp.41.11.04f

8. Capaldi CA, Liu L, Dopko RL. Santé mentale positive et changement perçu de la santé mentale chez les adultes au Canada pendant la deuxième vague de la pandémie de COVID-19. Promotion de la santé et prévention des maladies chroniques. 2021;41(11): 394-414. https://doi.org/10.24095 /hpcdp.41.11.05f

9. Liu L, Capaldi CA, Dopko RL. Idées suicidaires au Canada pendant la pandémie de COVID-19. Promotion de la santé et prévention des maladies chroniques. 2021;41(11):415-429. https://doi.org/10.24095/hpcdp.41.11 $.06 \mathrm{f}$

10. Gadermann AC, Thomson KC, Richardson CG, et al. Examining the impacts of the COVID-19 pandemic on family mental health in Canada: findings from a national cross-sectional study. BMJ Open. 2021;11(1): e042871. https://doi.org/10.1136 /bmjopen-2020-042871

11. Lee SJ, Ward KP, Lee JY, Rodriguez CM. Parental social isolation and child maltreatment risk during the COVID-19 pandemic. J Fam Violence. 2021;1-12. https://doi.org/10.1007 /s10896-020-00244-3

12. Cappa C, Jijon I. COVID-19 and violence against children: a review of early studies. Child Abuse Negl. 2021; 116(Pt 2):105053. https://doi.org/10 .1016/j.chiabu.2021.105053
13. Agence de la santé publique du Canada (ASPC). 2021-2022 Plan ministériel. Ottawa (Ont.) : ASPC; 2021 [No au catalogue : HP2-26F-PDF].

14. Gilbert R, Widom CS, Browne K, Fergusson D, Webb E, Janson S. Burden and consequences of child maltreatment in high-income countries. Lancet. 2009;373(9657):68-81. https://doi.org/10.1016/S0140-6736(08) 61706-7

15. Fore HH. Violence against children in the time of COVID-19: what we have learned, what remains unknown and the opportunities that lie ahead. Child Abuse Negl. 2021;116(Pt 2):104776. https://doi.org/10.1016/j.chiabu.2020 .104776

16. Katz I, Katz C, Andresen S, et al. Child maltreatment reports and child protection service responses during COVID-19: knowledge exchange among Australia, Brazil, Canada, Colombia, Germany, Israel, and South Africa. Child Abuse Negl. 2021;116(Pt 2): 105078. https://doi.org/10.1016/j.chiabu .2021 .105078

17. Baron EJ, Goldstein EG, Wallace CT. Suffering in silence: how COVID-19 school closures inhibit the reporting of child maltreatment. J Public Econ. 2020;190:104258. https://doi.org/10 .1016/j.jpubeco.2020.104258

18. Rapoport E, Reisert H, Schoeman E, Adesman A. Reporting of child maltreatment during the SARS-CoV-2 pandemic in New York City from March to May 2020. Child Abuse Negl. 2021;116(Pt 2):104719. https:// doi.org/10.1016/j.chiabu.2020.104719

19. Statistique Canada. Les affaires criminelles déclarées par la police diminuent au cours des premiers mois de la pandémie, alors que les demandes d'intervention liées à des querelles de ménage sont à la hausse. Ottawa (Ont.) : Le Quotidien; 1er sept. 2020 [consultation le 28 juin 2021]. En ligne à : https://www150.statcan.gc.ca/n1 /daily-quotidien/200901/dq200901a -fra.htm

20. Sharma S, Wong D, Schomberg J, et al. COVID-19: differences in sentinel injury and child abuse reporting during a pandemic. Child Abuse Negl. 2021;116(Pt 2):104990. https:// doi.org/10.1016/j.chiabu.2021.104990
21. Yousif N. 'Disturbing trend': Ottawa hospital sees rise in number of babies with severe head injuries during second wave of COVID-19 [Internet]. Toronto Star; 29 janv. 2021 [consultation le 29 juin 2021]. En ligne à : https://www.thestar.com/news/gta /2021/01/29/disturbing-trend-ottawa -hospital-sees-rise-in-number-of-babies -with-severe-head-injuries-during -second-wave-of-covid-19.html

22. Sidpra J, Abomeli D, Hameed B, Baker J, Mankad K. Rise in the incidence of abusive head trauma during the COVID-19 pandemic. Arch Dis Child. 2021;106(3):e14. https://doi.org /10.1136/archdischild-2020-319872

23. Connell CM, Strambler MJ. Experiences with COVID-19 stressors and parents' use of neglectful, harsh, and positive parenting practices in the Northeastern United States. Child Maltreat. 2021;26(3):25-66. https:// doi.org/10.1177/10775595211006465

24. Leatherdale ST, Manske S, Wong S, Cameron R. Integrating research, policy and practice in school-based physical activity prevention programming: the School Health Action, Planning and Evaluation System (SHAPES) Physical Activity Module. Health Promot Pract. 2009;10(2):254261. https://doi.org/10.1177/152483 9906298499

25. Tonmyr L, Mathews B, Shields ME, Hovdestad W, Afifi TO. Does mandatory reporting legislation increase contact with child protection? - a legal doctrinal review and an analytical examination. BMC Public Health. 2018;18(1):1021. https://doi.org/10 .1186/s12889-018-5864-0

26. McTavish JR, Gonzalez A, Santesso N, MacGregor JC, McKee C, MacMillan HL. Identifying children exposed to maltreatment: a systematic review update. BMC Pediatr. 2020;20(1):113. https://doi.org/10.1186/s12887-020 $-2015-4$

27. Agence de la santé publique du Canada. Arrêtons la violence familiale [Internet]. Ottawa (Ont.), ASPC; 2020 [consultation le 5 juill. 2021]. En ligne à : https://www.canada.ca/fr /sante-publique/services/promotion -sante/arretons-violence-familiale.html 
28. Agence de la santé publique du Canada (ASPC). Canada : un pays pionnier : feuille de route du Canada pour mettre fin à la violence à l'égard des enfants. Ottawa (Ont.) : ASPC; 2019 [No au catalogue : HP35-118/ 2019F-PDF]. En ligne à : https://www .canada.ca/content/dam/phac-aspc /documents/services/publications /healthy-living/road-map-end-violence -against-children/feuille-de-route-mettre -fin-violence-enfants.pdf

29. Commission de vérité et réconciliation du Canada (CVR). Commission de vérité et réconciliation du Canada : Appels à l'action. Winnipeg (Man.) : CVR; 2015. En ligne à : https:// ehprnh2mwo3.exactdn.com/wp-content /uploads/2021/04/4-Appels_a_l-Action _French.pdf

30. Leeb RT, Paulozzi L, Melanson C, Simon TR, Arias I. Child maltreatment surveillance: uniform definitions for public health and recommended data elements, Version 1.0. Atlanta (GA): Centers for Disease Control and Prevention, National Center for Injury Prevention and Control; 2008. En ligne à : https:// cdc.gov/violenceprevention/pdf/cm surveillance-a.pdf

31. Nsubuga P, White ME, Thacker SB, et al. Chapter 53. Public health surveillance: a tool for targeting and monitoring interventions. In: Jamison DT, Breman JG, Measham AR, et al., editors. Disease control priorities in developing countries. 2nd ed. Washington (DC): The International Bank for Reconstruction and Development / The World Bank; New York: Oxford University Press; 2006.

32. Chartier M, Nickel NC, Chateau D, et al. Families First Home Visiting programme reduces population-level child health and social inequities. J Epidemiol Community Health. 2018; 72(1):47-53. https://doi.org/10.1136 /jech-2017-209321

33. Organisation mondiale de la santé (OMS). The VPA approach: the public health approach [Internet]. Genève (Suisse) : OMS; [consultation le 4 oct. 2021]. En ligne à : https://www.who int/groups/violence-prevention-alliance /approach
34. Sumner SA, Mercy JA, Dahlberg LL, Hillis SD, Klevens J, Houry D. Violence in the United States: status, challenges, and opportunities. JAMA. 2015;314(5):478-488. https://doi.org /10.1001/jama.2015.8371

35. Australian Institute of Health and Welfare. Child protection Australia 2019-20. Child welfare series no. 74. Canberra (AU): AIHW; 2021 [AIHW Catalogue No. CWS 78]. https://doi .org/10.25816/g208-rp81

36. Campeau A, Qadri S, Barakat F, et collab. Aperçu - Cadre des indicateurs de surveillance de la maltraitance envers les enfants. Promotion de la santé et prévention des maladies chroniques au Canada 2020;40(2):5861. https://doi.org/10.24095/hpcdp.40 $.2 .04 \mathrm{f}$

37. Agence de la santé publique du Canada. Rapport de l'administrateur en chef de la santé publique sur l'état de la santé publique au Canada 2016 - Regard sur la violence familiale au Canada. Ottawa (Ont.) : ASPC; 2016. No au catalogue : HP2-1DF-PDF. 